preprint - UTF

\title{
Dirac Functional Determinants in Terms of the Eta Invariant and the Noncommutative Residue
}

\author{
Guido Cognola (1) $^{(1)}$, Emilio Elizalde $\phi^{(2)}$ and Sergio Zerbini $\left.\right|^{(1)}$ \\ ${ }^{(1)}$ Dipartimento di Fisica, Università di Trento \\ and Istituto Nazionale di Fisica Nucleare, \\ Gruppo Collegato di Trento, Italia \\ ${ }^{(2)}$ Consejo Superior de Investigaciones Científicas (CSIC) \\ Institut d'Estudis Espacials de Catalunya (IEEC) \\ Edifici Nexus 201, Gran Capità 2-4, 08034 Barcelona, Spain \\ and Departament ECM and IFAE, Facultat de Física, \\ Universitat de Barcelona, Diagonal 647, 08028 Barcelona, Spain
}

May 18, 2018

Abstract: The zeta and eta-functions associated with massless and massive Dirac operators, in a D-dimensional ( $D$ odd or even) manifold without boundary, are rigorously constructed. Several mathematical subtleties involved in this process are stressed, as the intrisic ambiguity present in the definition of the associated fermion functional determinant in the massless case and, also, the unavoidable presence (in some situations) of a multiplicative anomaly, that can be conveniently expressed in terms of the noncommutative residue. The ambiguity is here seen to disappear in the massive case, giving rise to a phase of the Dirac determinant - that agrees with very recent calculations appeared in the mathematical literature - and to a multiplicative anomaly — also in agreement with other calculations, in the coinciding situations. After explicit, nontrivial resummation of the mass series expansions involving zeta and eta functions, the results are expressed in terms of quite simple formulas.

PACS numbers: $\quad 02.30 . \mathrm{Tb}, 02.70 . \mathrm{Hm}, 04.62 .+\mathrm{V}$

Keywords: Zeta function-regularization, fermionic functional determinants, multiplicative anomaly, noncommutative residue, Wodzicki formula.

\footnotetext{
*e-mail: cognola@science.unitn.it

${ }^{\dagger}$ While on leave at: Department of Mathematics, Room 2-363A, Massachusetts Institute of Technology, 77 Massachusetts Av., Cambridge, MA 02139-4307, USA; e-mail: elizalde@ieec.fcr.es

${ }^{\ddagger} \mathrm{e}$-mail: zerbini@science.unitn.it
} 


\section{Introduction}

It is well known that, within the so-called one-loop approximation in Quantum Field Theory, the Euclidean one-loop effective action $\Gamma$ may be expressed in terms of the functional determinant of an elliptic differential operator $O$, defined on a $D$-dimensional manifold, namely

$$
\Gamma \equiv \ln \operatorname{det} O
$$

The ultraviolet one-loop divergences, which are present, may be regularized by means of the zeta-function regularization (for recent reviews, see [1, 2, 3]). In the bosonic sector, one is dealing, typically, with a second order self-adjoint non-negative operator $L$, whose spectrum is bounded from below (e.g., it is real and non-negative). In this case, a quite elementary approach is at our disposal, namely the heat-kernel technique, which allows to define the zeta-function in an elementary and direct way by means of the related Mellin transform, i.e.

$$
\zeta(s \mid L)=\frac{1}{\Gamma(s)} \int_{0}^{\infty} d t t^{s-1} \operatorname{Tr} e^{-t L},
$$

valid for $\operatorname{Re} s>D / 2$. Here $\operatorname{Tr} e^{-t L}=\sum_{i} e^{-t \lambda_{i}}, \lambda_{i}$ being the eigenvalues of $L$. As a consequence, $\zeta(s \mid L)=\sum_{i} \lambda_{i}^{-s}$. If zero modes are present, one has to subtract them, replacing $\operatorname{Tr} e^{-t L} \rightarrow$ $\operatorname{Tr} e^{-t L}-P_{0}, P_{0}$ being the projector onto the zero modes. The short- $t$ asymptotics of $\operatorname{Tr} e^{-t L}$ have been well studied both in the physical and in the mathematical literature [4, 5]. For a second-order operator on a boundaryless $D$-dimensional (smooth) manifold, it reads

$$
\operatorname{Tr} e^{-t L} \simeq \sum_{j=0}^{\infty} A_{j}(L) t^{j-D / 2},
$$

in which $A_{j}(L)$ are the Seeley-DeWitt coefficients, that can be computed with different techniques. Making use of the Mellin transform definition, a standard argument leads directly to a particular case of Seeley's meromorphic extension theorem, namely

$$
\zeta(s \mid L)=\frac{1}{\Gamma(s)}\left[\sum_{j=0}^{\infty} \frac{A_{j}(L)}{s+j-\frac{D}{2}}+J(s, u)\right],
$$

where $J(s, u)$ is the analytic part of the zeta-function. It turns out that this analytic extension is regular at $s=0$ and thus, the regularized functional determinant of $L$ can be defined by [6, 7, 8]

$$
\log \operatorname{det} L=-\zeta^{\prime}(0 \mid L)
$$

In the fermionic sector, the situation is quite different. Usually, one deals with a first order differential operator $A$ (the Dirac operator, the Rarita-Schwinger operator). To begin with, let us consider the self-adjoint case. Here the spectrum is unbounded over the whole real axis. It is obvious that heat-kernel techniques are no longer useful and one has to make use of the general theory of complex powers of a pseudo-differential operator ( $\Psi \mathrm{DO})$ [9]. In the following, we present some known facts about the complex powers of $\Psi$ DOs which we will often use.

Let us consider a $D$-dimensional, smooth (compact) manifold without boundary, $M_{D}$, and a (classical) $\Psi \mathrm{DO}, Q$, acting on sections of vector bundles on $M_{D}$. To any classical $\Psi \mathrm{DO}$, 
it corresponds a complete symbol $\sigma(Q)=Q(x, k)=e^{i k x} Q e^{-i k x}$, such that, modulo infinitely smoothing operators, one has

$$
(Q f)(x) \sim \int_{R^{D}} \frac{d k}{(2 \pi)^{D}} \int_{R^{D}} d y e^{i(x-y) k} Q(x, k) f(y) .
$$

The complete symbol of $Q$ admits an asymptotic expansion for $|k| \rightarrow \infty$, given by

$$
Q(x, k) \sim \sum_{j=0}^{\infty} Q_{q-j}(x, k),
$$

where the coefficients fulfill the homogeneity property $Q_{q-j}(x, t k)=t^{q-j} Q_{q-j}(x, k)$, for $t>0$, being $Q_{q}(x, k) \neq 0$. The number $q$ is called the order of $Q$ and $Q_{q}(x, k)$ its principal symbol. Recall that $Q$ is elliptic when $Q_{q}(x, k)$ is invertible.

The complex powers $Q_{\theta}^{-s}$ can be defined as soon as there exists a conical neighborough $C_{\theta}$ of a ray $L_{\theta}=\{\lambda \in C, \arg \lambda=\theta\}$ such that it contains, at most, only a finite number of eigenvalues of $Q$. Under this condition, the zeta function can be defined, for Re $s$ larger than an abscissa of convergence, given by $D / q$, as

$$
\zeta_{\theta}(s \mid Q)=\operatorname{Tr} Q_{\theta}^{-s}=\sum_{i} \lambda_{i}^{-s}
$$

where the sum is over the whole spectrum of $Q$ and $\lambda^{-s}$ is defined with respect to the admissible cut $L_{\theta}$, namely

$$
\lambda^{-s}=\exp \left(-s \ln _{\theta} \lambda\right), \quad \theta-2 \pi \leq \operatorname{Im}_{\ln } \lambda<\theta .
$$

It is possible to show again that $\zeta_{\theta}(s \mid Q)$ possesses a meromorphic continuation to the whole complex plane and that it is regular at the origin [9]. Thus, one can define

$$
\ln \operatorname{det} Q=-\zeta_{\theta}^{\prime}(0 \mid Q) \text {. }
$$

As a result, in general, both the zeta function as well as its derivative may depend on the spectral cut $L_{\theta}$. Following Wodzicki, one is led to study the function

$$
\rho(s \mid Q)=\zeta_{\theta}(s \mid Q)-\zeta_{\theta_{1}}(s \mid Q),
$$

where $L_{\theta_{1}}$ is another admissible cut. The general properties of $\rho(s \mid Q)$ have been investigated in [10, 11] and they can expressed by means of the noncommutative residue, that will be introduced in the next section. We shall mainly be interested in $\rho^{\prime}(0 \mid Q)$, since it is related to the ambiguity in the definition of the functional determinant of $Q$, and we will discuss it, in an elementary way. Here we only recall the case when the ambiguity may be present.

Let us introduce the two sectors of the complex plane associated with the two admissible cuts, namely

$$
S=\left\{\lambda, \theta<\arg \lambda<\theta_{1}\right\}, \quad S_{1}=\left\{\lambda, \theta_{1}-2 \pi<\arg \lambda<\theta\right\} .
$$

Now, it is possible to show that if there is an infinite number of eingenvalues of $Q$ in both sectors $S$ and $S_{1}$, then $\rho^{\prime}(0 \mid Q)$ is non-vanishing and there exists an ambiguity in the definition of the functional determinant of $Q$ [10, 11]. 
As shown by Wodzicki, a new spectral function plays a role in relation to this ambiguity. It is the eta-function of $Q$, introduced in Ref. [13], which, for large $\operatorname{Re} s$, is defined by

$$
\eta(s \mid Q)=\sum_{i} \operatorname{sign} \lambda_{i}\left|\lambda_{i}\right|^{-s}
$$

It can be proven that the eta-function has a meromorphic extension, which is regular at the origin [10, 11] .

If we apply these general results to the Dirac operator, it follows that, in general, there is an ambiguity in the definition of the effective action, already noticed in [14, 15], and the eta-invariant, i.e. $\eta(0 \mid Q)$, will enter the game. This happens only in odd dimension and it is known as the appearance of an intrisic parity anomaly term, the so called induced Chern-Simons term [16, 17]. In even dimension the ambiguity is still present, but depends on a Seeley-DeWitt coefficient and may be absorbed by a redefinition of the scale renormalization (see, for example [15]).

The contents of the paper are the following. In Sec. 2, some elements of Wodzicki's theory and the multiplicative anomaly are presented, for the sake of completeness. In Sec. 3, the zeta-function and the eta-function associated with the massless Dirac operator are revisited. An example is considered in detail and a brief study of the relevant Wodzicki function $\rho$ is presented. In Sec. 4 the massive case is considered and, specifically, the one-loop effective action in Sec. 5. The multiplicative anomaly corresponding to the very common case of the massive Dirac operator multiplied by its adjoint is obtained in Sec. 6. We also give here explicit formulas for the phase of the massive Dirac determinant and for its relation with that of the square root of the massive Laplacian. They constitute the main results of the paper. Some explicit examples are discussed in Sec. 7 in detail. Sec. 8 is devoted to conclusions. Finally, in an Appendix we analyze the disappearance of the ambiguity in the definition of the zeta function for the massive Dirac operator, determined by the sign of the mass term. This is also, in itself, a remarkable result.

\section{The noncommutative (or Wodzicki) residue}

For the reader's convenience, we will review in this section some information concerning the Wodzicki theory on the noncommutative residue [18] (see also [19, 10, 12, 11]) that will be used in the rest of the paper.

In order to introduce the definition of the noncommutative residue of a $\Psi D O, Q$, let us consider an elliptic positive (differential) operator $A$, with $a=2 p>q, p$ integer, and form the family of $\Psi$ DOs $A_{Q}(u)=A+u Q, u$ being a real parameter. The associated zeta-function reads

$$
\zeta\left(s \mid A_{Q}(u)\right)=\operatorname{Tr}(A+u Q)^{-s} .
$$

The meromorphic structure of the above zeta-function can be obtained from the short- $t$ asymptotics of $\operatorname{Tr} e^{-A_{Q}(u)}$ [20], namely

$$
\operatorname{Tr} e^{-t A_{Q}(u)} \simeq \sum_{j=1}^{\infty} \alpha_{j}(u) t^{(j-D) / 2 p}+\sum_{k=1}^{\infty} \beta_{k}(u) t^{k} \ln t .
$$


Note the presence of logarithmic terms that lead, using the Mellin transform, to double poles in the meromorphic expansion of $\zeta\left(s \mid A_{Q}(u)\right)$, i.e.

$$
\begin{aligned}
\zeta\left(s \mid A_{Q}(u)\right) & =\frac{1}{\Gamma(s)}\left(\int_{0}^{1}+\int_{1}^{\infty}\right) d t t^{s-1} \operatorname{Tr} e^{-A_{Q}(u)} \\
& =\frac{1}{\Gamma(s)}\left[\sum_{j=1}^{\infty} \frac{\alpha(u)_{j}}{s+\frac{j-D}{2 p}}-\sum_{k=1}^{\infty} \frac{\beta_{k}(u)}{(s+k)^{2}}+J(s, u)\right]
\end{aligned}
$$

where $J(s, u)$ is the analytic part. Taking the derivative with respect to $u$ and then the limit $u \rightarrow 0$, one gets

$$
\begin{aligned}
\lim _{u \rightarrow 0} \frac{d}{d u} \operatorname{Tr}(A+u Q)^{-s} & =-s \operatorname{Tr}\left(Q A^{-s-1}\right) \\
& =\frac{1}{\Gamma(s)}\left[\sum_{j=1}^{\infty} \frac{\alpha_{j}^{\prime}(0)}{s+\frac{j-D}{2 p}}-\sum_{k=1}^{\infty} \frac{\beta_{k}^{\prime}(0)}{(s+k)^{2}}+J^{\prime}(s, 0)\right] .
\end{aligned}
$$

By definition, the noncommutative residue of $Q$ is given by

$$
\operatorname{res}(Q)=\operatorname{Res}\left[2 p \lim _{u \rightarrow 0} \frac{d}{d u} \operatorname{Tr}(A+u Q)^{-s}\right]_{s=-1}=2 p \beta_{1}^{\prime}(0)
$$

where Res is the usual Cauchy residue. It is possible to show that $\operatorname{res}(Q)$ is independent on the elliptic operator $A$ and that it is a trace in the algebra of classical $\Psi D O$ s (actually, the only trace up to multiplicative constants). From the above definition and taking the derivative with respect to $u$ at $u=0$ of Eq. (2.2), one obtains a possible way to compute the noncommutative residue. In fact

$$
\operatorname{Tr}\left(Q e^{-t A}\right) \simeq-\sum_{j \neq D+2 p}^{\infty} \alpha_{j}^{\prime}(0) t^{(j-D) / 2 p-1}-\alpha_{D+2 p}^{\prime}-\frac{\operatorname{res}(Q)}{2 m} \ln t+O(t \ln t) .
$$

Thus, the noncommutative residue of $Q$ can be read off from the short $t$ asymptotics of the quantity $\operatorname{Tr}\left(Q e^{-t A}\right)$, just picking up the coefficient associated with $\ln t$.

When the manifold is non-compact, this is one of the methods that we have at hand for evaluating the Wodzicki residue, as long as all the traces involved exist. For the case of a compact manifold, Wodzicki has obtained a useful local form of the noncommutative residue, that is, a density which can be integrated to yield the noncommutative residue, namely

$$
\operatorname{res}(Q)=\int_{M_{D}} \frac{d x}{(2 \pi)^{D}} \int_{|k|=1} \operatorname{tr} Q_{-D}(x, k) d k .
$$

Here the component of order $-D$ (remember that $D$ is the dimension of the manifold) of the complete symbol appears as well as the internal trace tr. Eqs. (2.4) or (2.6) give

Lemma 1. In a neighborhood of $z=0$,

$$
\operatorname{Tr}\left(Q A^{-z}\right)=\frac{\operatorname{res}(Q)}{2 p z}+\frac{\gamma \operatorname{res}(Q)}{2 p}-\alpha_{D+2 p}^{\prime}(0)+O(z),
$$


where $\gamma$ is the Euler-Mascheroni constant. The latter equation gives another way to compute the noncommutative residue, namely (this expression was also found by Guillemin [12])

$$
\operatorname{res}(Q)=2 p\left[\operatorname{Res} \operatorname{Tr}\left(Q A^{-z}\right)\right]_{z=0} .
$$

If $A$ is a positive elliptic (differential) operator of positive order $a$, then we can write

$$
\zeta(A \mid s+\alpha)=\operatorname{Tr}\left[A^{-\alpha} A^{-s}\right]=\frac{\operatorname{res}\left(A^{-\alpha}\right)}{a s}+\text { finite terms }, \quad \alpha>0 .
$$

Thus, we see that the residues of the poles of the zeta function on the positive real axis are proportional to the noncommutative residues of some negative power of $A$. More precisely we obtain

$$
\left.\operatorname{Res} \zeta(A \mid s)\right|_{s=\alpha}=\frac{\operatorname{res}\left(A^{-\alpha}\right)}{a}, \quad \alpha>0 .
$$

With the latter formula we can compute the residues of all the poles situated on the positive real axis for the zeta function of any elliptic operator of positive order. Eq. (2.11) is more general than the well known and well used heat-kernel counterpart, since it works for operators of even order, as well as for operators of odd order (for examples and some applications see [22]).

We conclude this section recalling the multiplicative anomaly formula (see, for instance, [21, 23, 24, 25]). We consider two invertible, elliptic, positive (self-adjoint) operators, $A$ and $B$, on $M_{D}$ of positive orders, $a$ and $b$, respectively, and the quantity

$$
F(A, B)=\frac{\operatorname{det}(A B)}{(\operatorname{det} A)(\operatorname{det} B)}=e^{a(A, B)} .
$$

It is understood that the functional determinants are here defined by means of zeta-function regularization. By construction, $a(A, B)$ is called the multiplicative anomaly. We start from its expression in terms of the Kontsevich-Vishik multiplicative anomaly formula [21], namely

$$
a(A, B)=\int_{0}^{1} d t \operatorname{res}\left[\ln \left(A B^{-\frac{a}{b}}\right)\left(\frac{\ln A(t)}{a}-\frac{\ln [A(t) B]}{a+b}\right)\right] .
$$

This formula, Eq. (2.13), notably simplifies in the special case of commuting operators. In fact one then has

$$
a(A, B)=\frac{b}{2 a(a+b)} \operatorname{res}\left[\left(\ln \left(A B^{-\frac{a}{b}}\right)\right)^{2}\right]
$$

which can be rewritten as the Wodzicki multiplicative formula [19]

$$
a(A, B)=\frac{\operatorname{res}\left[\left(\ln \left(A^{b} B^{-a}\right)\right)^{2}\right]}{2 a b(a+b)}=a(B, A),
$$

where the symmetry property in $A$ and $B$ is manifest. Physical applications of this formula have been given in [23, 24, 25]. A direct computation of the multiplicative anomaly in higher dimensional manifolds can be found in [26] and an application to chiral anomaly has been given in [27]. Very recently, it has been shown that the multiplicative anomaly gives contributions to the one-loop effective action obtained by dimensional reduction 28. 


\section{The zeta function of the Dirac operator and its $\eta$ invariant}

As mentioned in the Introduction, if the elliptic operator $A$ is not positive definite, the general theory of complex powers of elliptic operators may still be used, but then another spectral function, namely the eta-function plays (besides the zeta function) a very important role. It is convenient to start with the assumption of dealing with a self-adjoint operator, which we will consider for the moment to be the massless Euclidean Dirac operator on a compact (curved) manifold $M$ of dimension $D$. It has the form

$$
A=i \not D=i \gamma^{\mu} \nabla_{\mu}, \quad \gamma_{\mu}=e_{k}^{\mu} \gamma^{k},
$$

where $e_{k}^{\mu}$ are "viel-bein" fields, $\gamma^{k}$ the Dirac matrices in $D$ dimensions and $\nabla_{\mu}$ the covariant derivative in the spin connection. Since $A^{+}=A$, the corresponding eigenvalues are real. Let us denote by $\lambda_{i}$ the positive eigenvalues and by $\mu_{i}$ the negative ones. Since the spectrum is contained in the whole real axis, we have essentially two possible definitions of zeta function, because there exist two essentially different cuts, one in the lower and the other in the upper half-planes respectively. In both cases, the associated sectors contain an infinite number of eigenvalues and the related zeta functions read (for $\operatorname{Re} s$ sufficiently large)

$$
\zeta_{+}(s \mid A)=\sum_{i} \lambda_{i}^{-s}+e^{-i \pi s} \sum_{i}\left(-\mu_{i}\right)^{-s}
$$

and

$$
\zeta_{-}(s \mid A)=\sum_{i} \lambda_{i}^{-s}+e^{i \pi s} \sum_{i}\left(-\mu_{i}\right)^{-s} .
$$

On the other hand, the eta-function may be also defined as

$$
\eta(s \mid A)=\sum_{i} \lambda_{i}^{-s}-\sum_{i}\left(-\mu_{i}\right)^{-s}=\operatorname{Tr}\left(\frac{A}{|A|}\left(A^{2}\right)^{-\frac{s}{2}}\right) .
$$

Making use of the above definitions, a direct computation gives [10]

$$
\eta(s \mid A)=\frac{1+e^{i \pi s}}{2 i \sin \pi s} \zeta_{+}(s \mid A)-\frac{1+e^{-i \pi s}}{2 i \sin \pi s} \zeta_{-}(s \mid A)
$$

and

$$
\zeta\left(\frac{s}{2} \mid A^{2}\right)=\frac{1-e^{-i \pi s}}{2 i \sin \pi s} \zeta_{-}(s \mid A)-\frac{1-e^{i \pi s}}{2 i \sin \pi s} \zeta_{+}(s \mid A),
$$

where $\zeta\left(z \mid A^{2}\right)$ is the zeta function associated with the spinor Laplacian $L=A^{2}$, a second order non negative self-adjoint elliptic operator, whose zeta function can be unambiguosly defined.

In order to study the Wodzicki function, namely

$$
\rho(s \mid A)=\zeta_{+}(s \mid A)-\zeta_{-}(s \mid A),
$$

we may express the two zeta functions in terms of the eta-function and $\zeta\left(z \mid A^{2}\right)$. From Eqs. (3.5) and (3.6), one gets

$$
\zeta_{ \pm}(s \mid A)=\frac{1}{2}\left[1+e^{\mp i \pi s}\right] \zeta\left(\frac{s}{2} \mid L\right)+\frac{1}{2}\left[1-e^{\mp i \pi s}\right] \eta(s \mid A) .
$$


As a consequence, we obtain the result

$$
\rho(s \mid A)=i \sin \pi s\left[\eta(s \mid A)-\zeta\left(\frac{s}{2} \mid L\right)\right] .
$$

For physical applications, it is also useful to give the first derivative with respect to $s$, i.e.

$$
\rho^{\prime}(s \mid A)=i \sin \pi s\left[\eta^{\prime}(s \mid A)-\frac{1}{2} \zeta^{\prime}\left(\frac{s}{2} \mid L\right)\right]+i \pi \cos \pi s\left[\eta(s \mid A)-\zeta\left(\frac{s}{2} \mid L\right)\right] .
$$

Then, the ambiguity is described by the value at $s=0$ of the Wodzicki function and its derivative. These values depend on the regularity at $s=0$ of $\eta(s \mid A)$ and $\zeta\left(\frac{s}{2} \mid L\right)$. The latter is known to be regular (the Seeley theorem). With regard to the eta-function, Wodzicki has proven its analyticity at the origin. However, in the particular but important case of the Dirac operator, a direct approach is known and we will use it.

First, let us consider the even dimensional case $D=2 p$. Here, the ' $\gamma^{5}$ ' matrix exists, namely: $\gamma^{D+1}=\gamma^{1} \cdots \gamma^{D}$, and, as is well known, since $\gamma^{D+1}$ anticommutes with $\not D$, both $\lambda_{i}$ and $-\lambda_{i}$ are eigenvalues for $\not D$ with eigenfunctions $\psi_{i}^{+}$and $\psi_{i}^{-}=\gamma^{D+1} \psi_{i}^{+}$respectively. In these conditions, the eta-function identically vanishes. We have $\rho(0 \mid A)=0$, and

$$
\begin{gathered}
\zeta_{ \pm}(s \mid A)=\frac{1}{2}\left[1+e^{\mp i \pi s}\right] \zeta\left(\frac{s}{2} \mid L\right), \\
\zeta_{+}(0 \mid A)=\zeta_{-}(0 \mid A)=A_{p}(L), \\
\zeta_{ \pm}^{\prime}(0 \mid A)=\frac{1}{2} \zeta^{\prime}(0 \mid L) \mp \frac{i \pi}{2} A_{p}(L),
\end{gathered}
$$

and

$$
\zeta_{+}^{\prime}(0 \mid A)-\zeta_{-}^{\prime}(0 \mid A)=-i \pi A_{p}(L) .
$$

Here the ambiguity depends on the local functional of the external gauge field, the Seeley-DeWitt coefficient $A_{p}(L)$, and since the one-loop effective action is defined modulo counterterms of the same nature, it can be reabsorbed by the renormalization procedure.

In the odd dimensional case $D=2 p+1$ however, there is no symmetry in the spectrum and one has the possibility of a non trivial eta-function. Furthermore, starting from (3.4), one also may use the Mellin representation of the eta-function, namely for Re $s$ sufficiently large

$$
\eta(s \mid A)=\frac{1}{\Gamma(s)} \int_{0}^{\infty} d t t^{s-1} \operatorname{Tr}\left(\frac{A}{|A|} e^{-t|A|}\right),
$$

which can be conveniently rewritten as

$$
\eta(s \mid A)=\frac{1}{\Gamma\left(\frac{s+1}{2}\right)} \int_{0}^{\infty} d t t^{\frac{s+1}{2}-1} \operatorname{Tr}\left(A e^{-t A^{2}}\right) .
$$

By the standard heat kernel techniques, we can try to find the analytic continuation of the eta-invariant. To this aim, one needs the short $t$ asymptotics of the integrand. This involves a 
highly non trivial calculation and the answer is provided by a theorem due to Bismut and Freed [29, which states that $\operatorname{Tr}\left(A e^{-t A^{2}}\right)=O\left(t^{1 / 2}\right)$. More precisely

$$
\operatorname{Tr}\left(A e^{-t A^{2}}\right) \simeq \sum_{l=0}^{\infty} C_{l+p+2} t^{l+\frac{1}{2}},
$$

where the $C_{r}$ are suitable coefficients. Thus, the merophormic extension reads

$$
\eta(s \mid A)=\frac{1}{\Gamma\left(\frac{s+1}{2}\right)} \sum_{r=0}^{\infty} \frac{C_{l+p+2}}{s / 2+l+1}+\eta_{0}(s \mid A),
$$

from which the regularity at $s=0$ of $\eta(s \mid A)$ directly follows. In the latter equation, $\eta_{0}(s \mid A)$ is an analytic function of $s$. As a result,

$$
\begin{gathered}
\zeta_{+}(0 \mid A)=\zeta_{-}(0 \mid A)=0 \\
\zeta_{ \pm}^{\prime}(0 \mid A)=\frac{1}{2} \zeta^{\prime}(0 \mid L) \pm \frac{i \pi}{2} \eta(0 \mid A)
\end{gathered}
$$

and

$$
\zeta_{+}^{\prime}(0 \mid A)-\zeta_{-}^{\prime}(0 \mid A)=i \pi \eta(0 \mid A)
$$

Here, the ambiguity is non-trivial, since, as it is clear from (3.18), $\eta(0 \mid A)$ is a non local functional of the external gauge field and it cannot be removed by addition of counterterms. Note that, in fact, all our results are particular cases of Wodzicki's main theorem and its corollaries and we need no new proofs. The originality resides in that we have made them absolutely explicit and applicable to the very important case of the physics of the Dirac operator.

Futhermore, from the Bismut and Freed theorem, and making a Mellin transform inversion, we also get

$$
\operatorname{Tr}\left(\frac{A}{|A|} e^{-t|A|}\right)=\frac{1}{2 \pi i} \int_{\operatorname{Re} z>0} d z t^{-z} \Gamma(z) \eta(z \mid A) .
$$

For $z=0$, one has a simple pole, while for $z=-2,-4, .$. , one has double poles. Shifting the vertical line to the left, one obtains the short $t$ asymptotics

$$
\begin{aligned}
\operatorname{Tr}\left(\frac{A}{|A|} e^{-t|A|}\right) \simeq & \eta(0 \mid A) \\
& +\sum_{l=0}^{\infty} \frac{2 C_{l+2+p}}{(2 l+2) ! \Gamma\left(-l-\frac{1}{2}\right)}\left[\gamma-\Psi\left(-l-\frac{1}{2}\right)-\ln t\right] t^{2 l+2}
\end{aligned}
$$

where $\Psi(z)$ is the di-gamma function. It should be noted the presence of logarithmic terms in this short- $t$ expansion, due to the fact that one is dealing with a $\Psi$ DO. From Eq. (3.23) we have

$$
\lim _{t \rightarrow 0} \operatorname{Tr}\left(\frac{A}{|A|} e^{-t|A|}\right)=\eta(0 \mid A)
$$


which may be useful in the evaluation of the eta invariant. Another representation of the eta invariant, which easily follows, is

$$
\eta(0 \mid A)=\int_{0}^{\infty} d t \operatorname{Tr}\left(A e^{-t|A|}\right) .
$$

As an application of Eq. (3.24), we present a simple proof of the classical result obtained in [17], where quantum massless fermions coupled to a classical gauge field in $2+1$ dimensions were shown to generate a Chern-Simons term, confirming the result got in [30] within a local zeta-function approach. The idea is to compute the first variation of the eta-invariant. Starting from Eq. (3.24), one gets

$$
\delta \eta(0 \mid A)=-\lim _{t \rightarrow 0}\left[t \operatorname{Tr}\left(\delta A e^{-t|A|}\right)\right] .
$$

Here $\delta A=\delta\left(i \gamma^{\mu} \partial_{\mu}-e \gamma^{\mu} A_{\mu}\right)=-e \gamma^{\mu} \delta A_{\mu}, A_{\mu}$ being the electromagnetic potential. Thus,

$$
\operatorname{Tr}\left(\delta A e^{-t|A|}\right)=-e \int d x \delta A_{\mu} \operatorname{tr}\left(\gamma^{\mu} e^{-t|A|}(x, x)\right) .
$$

The spectral theorem gives

$$
e^{-t|A|}(x, x)=\int_{0}^{\infty} d \lambda e^{-t \sqrt{\lambda}} \rho_{\lambda}\left(A^{2}\right)(x, x) .
$$

Since one is interested in the $t \rightarrow 0$ limit, only the asymptotics for $\lambda \rightarrow \infty$ of the local spectral density is relevant. In $D=3$, one has (see, for example, [31]) when $\lambda \rightarrow \infty$

$$
\rho_{\lambda}\left(A^{2}\right)(x, x) \simeq \frac{\lambda^{\frac{1}{2}}}{\Gamma\left(\frac{3}{2}\right)}+a_{1}(x) \frac{\lambda^{-\frac{1}{2}}}{\Gamma\left(\frac{1}{2}\right)}+O\left(\lambda^{-\frac{3}{2}}\right),
$$

where $a_{1}(x)=\frac{\gamma^{\nu} F_{\nu}^{*}}{(4 \pi)^{3 / 2}}$, with $F_{\mu}=\varepsilon_{\mu \nu \rho} F^{\nu \rho}$, is the first non-trivial Seeley-DeWitt coefficient releted to the spinor Laplacian. The leading term gives no contribution and taking the internal traces, one has for the non trivial ground state fermion current contribution

$$
\frac{\delta \eta(0 \mid A)}{\delta A_{\mu}}=-\frac{e}{2 \pi^{2}} F_{\mu}^{*},
$$

which is the Redlich result. We note that the sign ambiguity is always present in the massless case, because the total effective action is given by $(3.20)$ and has nothing to do with the PauliVillars regularization, as often claimed in the literature.

We close this section by presenting a heuristic argument for the regularity of the eta-function at the origin. Let us start from (3.4), namely

$$
\eta(s \mid A)=\operatorname{Tr}\left(Q\left(A^{2}\right)^{-\frac{s}{2}}\right),
$$

with $Q=\frac{A}{|A|},|A|=\sqrt{A^{2}}$. $Q$ is a $\Psi$ DO of zero order. By making use of Lemma 1, near the origin we have

$$
\eta(s \mid A)=\frac{\operatorname{res}(Q)}{s}+\frac{\gamma}{2} \operatorname{res}(Q)-\alpha_{D+2}^{\prime}(0)+O(s) .
$$

Thus, we have arrived at the following statement: 
Proposition 1. The eta-function related to a non positive elliptic operator $A$ is regular at $s=0$ if and only if $\operatorname{res}\left(\frac{A}{|A|}\right)=0$, where $|A|=\sqrt{A^{2}}$.

Example 1. On a compact flat manifold $M$ with odd dimension $D$, the massless Dirac operator has the form

$$
A=i \not D=i \gamma^{\mu} \nabla_{\mu}
$$

$\gamma^{\mu}$ being the Dirac matrices in $D-1$ dimensions. For the sake of simplicity, let us work in $D=3$ and assume a constant gauge potential $V_{\mu}$, namely a vanishing field strenght $F_{\mu \nu}$. Thus

$$
A^{2}=-\not D^{2}=-\square, \quad|A|=\sqrt{-\square} .
$$

The local symbol reads

$$
\sigma\left(\frac{A}{|A|}\right)(x, k)=\frac{\not k+\not}{\sqrt{k^{2}+V^{2}+2 \not k X}}
$$

In order to select the homogeneous component of degree -3 , we may consider $k \rightarrow \lambda k$, with $\lambda$ very large, and pick up the term proportional to $1 / \lambda^{3}$. One gets, with $|k|=1$,

$$
\sigma\left(\frac{A}{|A|}\right)(x, \lambda k)=\ldots+\left(C_{1} \not+C_{2}(\not)^{3}\right) \lambda^{-3}+\ldots
$$

where the $C_{1}$ and $C_{2}$ are simple functions of $V$. In the evaluation of the Wodzicki residue one has to take the internal trace tr and this gives a vanishing contribution because, in $D=3$, not only $\operatorname{tr} X=0$, but also $\operatorname{tr}\left(X^{3}\right)=0$, since $\operatorname{tr} \gamma_{\mu} \gamma_{\nu} \gamma_{\rho}=2 \varepsilon_{\mu \nu \rho}$.

\section{The massive Dirac operator}

Let us consider now the case of massive spinors. Denoting the massive operator by $K=A+i m$, we have

$$
K \psi_{i}=(A+i m) \psi_{i}=\left(\lambda_{i}+i m\right) \psi_{i}
$$

where, as above, $\lambda_{i}$ and $\psi_{i}$ are the eigenvalues and eigenfunctions of $A . K$ is non-hermitian and its eigenvalues are now complex numbers. As in the massless case, we also have here two possible spectral cuts, and the two zeta functions may in principle be defined as

$$
\zeta_{ \pm}(s \mid K)=\sum_{\lambda_{i}>0}\left(\lambda_{i}+i m\right)^{-s}+\left.\sum_{\mu_{i}<0}\left[e^{\mp i \theta}\left(-\mu_{i}\right)+i m\right]^{-s}\right|_{\theta=\pi} .
$$

However, due to the presence here of the mass term $+i m$ (and being the mass $m$ positive), in order to avoid a singularity in the complex powers of the spectrum of $K=A+i m$ when we perform the analytical continuation of the spectral values (from positive $-\mu_{i}$ to negative $\mu_{i}$, along a path $\left.\Gamma_{ \pm}:-\mu_{i} e^{ \pm i \theta}, 0 \leq \theta \leq \pi\right)$, it is compulsory to take $\zeta_{+}(s \mid K)$ - e.g., to perform the analytic continuation in the upper half plane. In fact, a singular point is lying in the lower half complex $\mu$-plane, namely at -im, that gives rise to a cut in the complex $s$-plane when performing then the analytic continuation in $s$. Note that we have to perform two different analytical continuations 
here, in a compatible way, and this is actually guaranteed in our method (see a more detailed discussion of this issue in the Appendix). Quite on the contrary, when we will consider, below, the adjoint operator $K^{+}=A-i m$, the analytical continuation must be performed on the lower half-plane, namely the relevant zeta function will be $\zeta_{-}\left(s \mid K^{+}\right)$, in order to avoid the singularity (see also the Appendix). As we shall prove in a subsequent section, this argument turns out to be very important for the obtaintion of the correct value for the multiplicative anomaly corresponding to the product of the two operators, $K$ and $K^{+}$. Notice also that our definition of the zeta function for the operators $K$ and $K^{+}$slightly differs from the one adopted in [15], where the imaginary part of the eigenvalues was also rotated. T We believe that our definition is the most natural one in the massive case, being the minimal extension of the corresponding definition for selfadjoint operators with (in general) positive and negative spectrum - that was on its turn obtained from the rigorous mathematical theory for elliptic selfadjoint operators, with no negative eigenvalues.

If the mass is sufficiently small, binomial expansion gives

$$
\zeta_{+}(s \mid K)=\sum_{k=0}^{\infty} \frac{(-1)^{k} \Gamma(s+k)(i m)^{k}}{\Gamma(s) k !} \zeta_{+}(s+k \mid A),
$$

and analogously for $\zeta_{-}\left(s \mid K^{+}\right)$. Making use of Eqs. (3.8), one arrives at

$$
\begin{gathered}
\zeta_{+}(s \mid K)=\frac{1}{2}\left[1+e^{-i \pi s}\right] \zeta\left(\frac{s}{2} \mid L\right)+\frac{1}{2}\left[1-e^{-i \pi s}\right] \eta(s \mid A) \\
+\sum_{k=1}^{\infty} \frac{(-1)^{k} \Gamma(s+k)(i m)^{k}}{2 \Gamma(s) k !}\left\{\left[1+e^{-i \pi(s+k)}\right] \zeta\left(\frac{s+k}{2} \mid L\right)\right. \\
\left.\quad+\left[1-e^{-i \pi(s+k)}\right] \eta(s+k \mid A)\right\}
\end{gathered}
$$

We have thus obtained, in a direct way, the meromorphic extension of the zeta function associated with the massive Dirac operator, starting from the knowledge of the massless one.

As an example, if $M$ is a flat even-dimensional manifold, then $\zeta(s \mid L)$ has only a simple pole at $s=D / 2$ with residue equal to $(4 \pi)^{-D / 2} D \mathcal{V}, \mathcal{V}$ being the volume of $M$. This means that $\zeta(s \mid K)$ has a finite number of simple poles at the points $s=D-k>0(k=0,1, \ldots, D-1)$, whose residues read

$$
\left.\operatorname{Res} \zeta_{+}(s \mid K)\right|_{s=D-k>0}=\frac{2 \mathcal{V} \Gamma(D+1)(-i m)^{k}}{(4 \pi)^{D / 2} \Gamma(D / 2) \Gamma(D-k) k !} .
$$

Observe that this result is independent of the choice of definition for the zeta function $\left(\zeta_{ \pm}\right)$.

Furthermore, consider the free massive Dirac operator in a flat manifold. For the symbol, we have

$$
\sigma(\not D D+i m)=\not p+i m, \quad \sigma(\not D D+i m)^{-\alpha}=\frac{1}{\not^{\alpha}}\left[\sum_{k=0}^{\infty}\left(\frac{-i m}{\not p^{\prime}}\right)^{k}\right]^{\alpha}
$$

and, in order to compute the non-commutative residue, we must pick up the coefficient of $\not{ }^{-D}$ in the previous expansion. We see that such a coefficient is non-vanishing only when $\alpha=n$ is

\footnotetext{
${ }^{4}$ To compare, the definition in [15] corresponds to rotating $\left(-\mu_{i}-i m\right) e^{ \pm i \theta}, 0 \leq \theta \leq \pi$, from $\theta=0$ to $\theta=\pi$.
} 
an integer number smaller than or equal to $D$. Under such condition, we get

$$
\operatorname{res}\left([A+i m]^{-n}\right)=\frac{\Gamma(D+1)(-i m)^{D-n}}{(4 \pi)^{D / 2} \Gamma(D / 2) \Gamma(n)(D-n) !} .
$$

Using this result in Eq. (2.7) we obtain Eq. (4.5) again.

\section{The one-loop effective action}

In this section we will compute the effective action for a massive spinor field on a $D$-dimensional manifold in terms of the zeta-function and the eta-function of the related massless operator. The effective action turns out to be proportional to the logarithm of the functional determinant of the Dirac operator, which may be expressed as minus the first derivative of the zeta function at zero. The massive Dirac operator $K=A+i m$ is non-hermitian, and one has $K^{+}=A-i m=K-2 i m$. However, $K^{+} K=L+m^{2}$ is a positive self-adjoint second order Laplace-like operator, and binomial expansion gives

$$
\zeta\left(s \mid K^{+} K\right)=\zeta\left(s \mid L+m^{2}\right)=\zeta(s \mid L)+\sum_{j=1}^{\infty}(-1)^{j} \frac{\Gamma(s+j) m^{2 j}}{\Gamma(s) j !} \zeta(s+j \mid L) .
$$

It is plain that the functional determinant associated with $L+m^{2}$ will play here an important role.

Let us begin with the even dimensional case, i.e. $D=2 p$. Recall that, in this situation, $\eta(s \mid A)=0$. Splitting the sum in Eq. (4.4) into even and odd terms and making use of Eqs. (3.8), it is easy to show that the latter gives a contribution of order $O\left(s^{2}\right)$. We can isolate the singular term by writing

$$
\zeta_{+}(s \mid K)=\frac{1}{2}\left[1+e^{-i \pi s}\right] \zeta\left(\frac{s}{2} \mid L\right)+\frac{1}{\Gamma(s)}\left(\frac{G_{+}(s)}{s}+\phi_{+}(s)\right)+O\left(s^{2}\right),
$$

and

$$
\zeta_{-}\left(s \mid K^{+}\right)=\frac{1}{2}\left[1+e^{i \pi s}\right] \zeta\left(\frac{s}{2} \mid L\right)+\frac{1}{\Gamma(s)}\left(\frac{G_{-}(s)}{s}+\phi_{-}(s)\right)+O\left(s^{2}\right),
$$

where

$$
\begin{array}{r}
\phi_{ \pm}(s)=\frac{1}{2} \sum_{j=1}^{p} \frac{(-1)^{j} \Gamma(s+2 j) m^{2 j}}{(2 j) !}\left[1+e^{\mp i \pi s}\right]\left[\zeta\left(\frac{s}{2}+j \mid L\right)-\frac{2 A_{p-j}(L)}{s \Gamma\left(\frac{s}{2}+j\right)}\right] \\
+\frac{1}{2} \sum_{j=p+1}^{\infty} \frac{(-1)^{j} \Gamma(s+2 j) m^{2 j}}{(2 j) !}\left[1+e^{\mp i \pi s}\right] \zeta\left(\frac{s}{2}+j \mid L\right)
\end{array}
$$

and

$$
G_{ \pm}(s)=\sum_{j=1}^{p} \frac{(-1)^{j} \Gamma(s+2 j) m^{2 j}}{(2 j) !}\left[1+e^{\mp i \pi s}\right] \frac{A_{p-j}(L)}{\Gamma\left(\frac{s}{2}+j\right)} .
$$

It is easy to see that both $\phi_{ \pm}(s)$ are regular at $s=0$. Taking derivatives at $s=0$, we get

$$
\zeta_{+}^{\prime}(0 \mid K)=\frac{1}{2}\left[\zeta^{\prime}(0 \mid L)-i \pi \zeta(0 \mid L)\right]+\gamma G_{+}(0)+G_{+}^{\prime}(0)+\phi_{+}(0),
$$


and a similar expression for $\zeta_{-}^{\prime}\left(0 \mid K^{+}\right)$.

On the other hand, from (5.1), one readily has

$$
\frac{1}{2} \zeta^{\prime}\left(0 \mid L+m^{2}\right)=\frac{1}{2} \zeta^{\prime}(0 \mid L)+\gamma G(0)+\phi(0)
$$

where $G(0)=G_{ \pm}(0)$ and analogously $\phi(0)=\phi_{ \pm}(0)$. Comparing Eqs. (5.6) and (5.7) we finally obtain

$$
\zeta_{+}^{\prime}(0 \mid K)=\frac{1}{2}\left[\zeta^{\prime}\left(0 \mid L+m^{2}\right)-i \pi \zeta\left(0 \mid L+m^{2}\right)\right]+\sum_{j=1}^{p} \frac{(-1)^{j} c_{j} m^{2 j} A_{p-j}(L)}{j !}
$$

and

$$
\zeta_{-}^{\prime}\left(0 \mid K^{+}\right)=\frac{1}{2}\left[\zeta^{\prime}\left(0 \mid L+m^{2}\right)+i \pi \zeta\left(0 \mid L+m^{2}\right)\right]+\sum_{j=1}^{p} \frac{(-1)^{j} c_{j} m^{2 j} A_{p-j}(L)}{j !}
$$

with

$$
c_{j}=\sum_{l=1}^{j} \frac{1}{2 l-1} .
$$

The above formula may be useful in the actual evaluation of the derivative of the zeta function of the massive Dirac operator. Here, again, the ambiguity depends on the Seeley-DeWitt coefficient, $\zeta\left(0 \mid L+m^{2}\right)$, only. We should point out that, as it stands, the above equation is valid for arbitrary, but finite, values of the mass $m$. This observation allows us to obtain a large mass expansion, simply by recalling an asymptotic theorem due to Voros [32], which states that, for large $m$, the asymptotics of the massive Laplacian are determined by the short asymptotics of its heat-kernel trace. As a result, we have

$$
\zeta\left(s \mid L+m^{2}\right) \simeq \sum_{r=0}^{\infty} \frac{A_{r}(L) \Gamma(r-p+s)}{\Gamma(s)} m^{2(p-r-s)} \simeq \sum_{r=0}^{p} \frac{(-1)^{p-r}}{(p-r) !} A_{r} m^{2 p-2 r-2 s}+O(s),
$$

and

$$
\begin{gathered}
\zeta^{\prime}\left(0 \mid L+m^{2}\right) \simeq \sum_{r=0}^{p-1} \frac{(-1)^{p-r} A_{r}(L)}{(p-r) !} m^{2(p-r)}\left(-\ln m^{2}+\sum_{j=1}^{p-r} \frac{1}{j}\right)-A_{p} \ln m^{2} \\
+\sum_{r=p+1}^{\infty} A_{r}(L) \Gamma(r-p) m^{2(p-r)}
\end{gathered}
$$

To summarize, we have reduced the problem of the first order masiive Dirac Operator (in the even dimensional case) to the much more familiar one for the simple Laplacian operator $L+m^{2}$, and traced back the terms coming from the previous ambiguities (fixed for $m \neq 0$ ) of the zeta function definition, to the term involving the Seeley-DeWitt coefficient $\zeta\left(0 \mid L+m^{2}\right)$. Our results are in agreement with the ones obtained in [15], with a completely different method. 
In the odd dimensional case, namely $D=2 p+1$, the eta invariant is non-vanishing and we have to refer to its meromorphic continuation given by the Eq. (3.18). As a consequence,

$$
\begin{aligned}
\zeta_{+}^{\prime}(0 \mid K)= & \frac{1}{2} \zeta^{\prime}(0 \mid L)+\frac{i \pi}{2} \eta(0 \mid A)+\sum_{j=1}^{\infty} \frac{(i m)^{2 j}}{2 j} \zeta(j \mid L) \\
& -\sum_{j=0}^{\infty} \frac{(i m)^{2 j+1}}{2 j+1} \eta(2 j+1 \mid A)+\frac{i \pi}{2} \sum_{j=0}^{p} \frac{(i m)^{2 j+1}}{\Gamma\left(j+\frac{3}{2}\right)} A_{p-j}(L),
\end{aligned}
$$

and

$$
\begin{aligned}
\zeta_{-}^{\prime}\left(0 \mid K^{+}\right)= & \frac{1}{2} \zeta^{\prime}(0 \mid L)-\frac{i \pi}{2} \eta(0 \mid A)+\sum_{j=1}^{\infty} \frac{(i m)^{2 j}}{2 j} \zeta(j \mid L) \\
& -\sum_{j=0}^{\infty} \frac{(-i m)^{2 j+1}}{2 j+1} \eta(2 j+1 \mid A)-\frac{i \pi}{2} \sum_{j=0}^{p} \frac{(i m)^{2 j+1}}{\Gamma\left(j+\frac{3}{2}\right)} A_{p-j}(L) .
\end{aligned}
$$

If we introduce the eta-like functions

$$
\eta(s \mid A \pm i m)=\sum_{i}\left(\lambda_{i} \pm i m\right)^{-s}-\sum_{i}\left(-\mu_{i} \pm i m\right)^{-s},
$$

for small mass, we have

$$
\eta(s \mid A \pm i m)=\eta(s \mid A)+\sum_{k=1}^{\infty} \frac{(\mp i m)^{k} \Gamma(s+k)}{\Gamma(s) k !} \eta(s+k \mid A) .
$$

Thus, the meromorphic properties of $\eta(s \mid A \pm i m)$ follow from the ones of the eta function. In particular, one obtains

$$
\eta^{\prime}(0 \mid A+i m)-\eta^{\prime}(0 \mid A-i m)=-2 \sum_{j=0}^{\infty} \frac{(i m)^{2 j+1}}{2 j+1} \eta(2 j+1 \mid A) .
$$

As a result, we have

$$
\begin{aligned}
\zeta_{+}^{\prime}(0 \mid K)= & \frac{1}{2} \zeta^{\prime}\left(0 \mid L+m^{2}\right)+\frac{i \pi}{2} \eta(0 \mid A) \\
& +\frac{1}{2}\left[\eta^{\prime}(0 \mid A+i m)-\eta^{\prime}(0 \mid A-i m)\right]-\frac{\pi}{2} \sum_{j=0}^{p} \frac{(-1)^{j} m^{2 j+1}}{\Gamma\left(j+\frac{3}{2}\right)} A_{p-j}(L),
\end{aligned}
$$

and

$$
\begin{aligned}
\zeta_{-}^{\prime}\left(0 \mid K^{+}\right)= & \frac{1}{2} \zeta^{\prime}\left(0 \mid L+m^{2}\right)-\frac{i \pi}{2} \eta(0 \mid A) \\
& -\frac{1}{2}\left[\eta^{\prime}(0 \mid A+i m)-\eta^{\prime}(0 \mid A-i m)\right]+\frac{\pi}{2} \sum_{j=0}^{p} \frac{(-1)^{j} m^{2 j+1}}{\Gamma\left(j+\frac{3}{2}\right)} A_{p-j}(L) .
\end{aligned}
$$

It should be noted the appearance of other two "eta-like" non local contributions. This, again, is valid for arbitrary value of the mass $m$. Also in the odd dimensional case, there is 
agreement with the expressions obtained in [15], even though, in those papers, the extra nonlocal contributions appeared in a different form. Eq. (3.15) leads to

$$
\eta^{\prime}(0 \mid A+i m)-\eta^{\prime}(0 \mid A-i m)=-2 i \int_{0}^{\infty} \frac{\sin m t}{t} \operatorname{Tr}\left(\frac{A}{|A|} e^{-t|A|}\right) .
$$

With regard to the large mass limit of the non-local contributions, we observe that, from the Voros theorem, one has

$$
\zeta^{\prime}\left(0 \mid L+m^{2}\right) \simeq \sum_{r=0}^{\infty} \Gamma\left(r-p-\frac{1}{2}\right) A_{r}(L) m^{2 p-2 r+1}
$$

while, from Eqs. (3.24) and (5.20),

$$
\lim _{m \rightarrow \infty}\left[\eta^{\prime}(0 \mid A+i m)-\eta^{\prime}(0 \mid A-i m)\right]=-i \pi \eta(0 \mid A)
$$

easily follows. This means that in the large mass limit the leading eta contribution cancel and we finally obtain

$$
\zeta_{+}^{\prime}(0 \mid K) \sim \frac{1}{2} \sum_{j=0}^{p}\left[\Gamma\left(-j-\frac{1}{2}\right)-\frac{(-1)^{j} \pi}{\Gamma\left(j+\frac{3}{2}\right)}\right] A_{p-j}(L) m^{2 j+1}+O(1 / m) .
$$

\section{The multiplicative anomaly for the massive Dirac operator}

If four dimensions it is usually assumed that $\operatorname{det} K=\operatorname{det} K^{+}=\sqrt{\operatorname{det} K^{+} K}$ (see, for example, the recent papers [33]). However, a multiplicative anomaly may be present sometimes, what will expoil the second equality, while the first one could be spoiled by a phase of the determinant. This has been recently discussed in detail in [34], where a very simple, specific example in which this situation occurs has been given (see also the contribution [35]).

To begin with, it should be noted here that, strictly speaking, the Wodzicki multiplicative anomaly formula is not valid for Dirac-like operators, since they are (first order) non positive operators. However, we still may apply the direct definition of the multiplicative anomaly, i.e.

$$
a\left(K^{+}, K\right)=\ln \operatorname{det} K^{+} K-\ln \operatorname{det} K^{+}-\ln \operatorname{det} K .
$$

The determinant of the differential operator $K^{+} K=L+m^{2}$ is defined through the zeta function by

$$
-\ln \operatorname{det} K^{+} K=-\ln \operatorname{det}\left(L+m^{2}\right)=\zeta^{\prime}\left(0 \mid L+m^{2}\right),
$$

while for $K$ and $K^{+}$themselves, we have to set

$$
-\ln \operatorname{det} K=\zeta_{+}^{\prime}(0 \mid K), \quad-\ln \operatorname{det} K^{+}=\zeta_{-}^{\prime}\left(0 \mid K^{+}\right) .
$$

The choice $\zeta_{+}$and $\zeta_{-}$for $K$ and $K^{+}$respectively is a consequence of the discussion we have carried out at the beginning of the previous section. Now, we have

Proposition 2. For the regularized determinant one gets the nice property

$$
\ln \operatorname{det} K^{+}=(\ln \operatorname{det} K)^{*} \text {, }
$$

which is valid for the determinant of non-hermitian matrices. 
Coming back to the multiplicative anomaly, we obtain from Eqs. (6.3), (5.8) and (5.9) a non vanishing result, indeed

Proposition 3. The multiplicative anomaly for the massive Dirac operator and its adjoint, in a space of even dimension $D=2 p$, reads

$$
a_{2 p}\left(K^{+}, K\right)=2 \sum_{j=1}^{p} \frac{(-1)^{j} m^{2 j} c_{j}}{j !} A_{p-j}(L) .
$$

Proposition 4. The multiplicative anomaly for the massive Dirac operator and its adjoint, in a space of odd dimension $D=2 p+1$, is given by

$$
a_{2 p+1}\left(K^{+}, K\right)=\pi \sum_{j=0}^{p}(-1)^{j+1} \frac{m^{2 j+1}}{\Gamma\left(j+\frac{3}{2}\right)} A_{p-j}(L) .
$$

$A_{p-j}(L)$ are the Seeley-DeWitt coefficients corresponding to the operator $L$. Of course, when $m \rightarrow 0$ the multiplicative anomaly vanishes. Directly in terms of the determinants of the Dirac operators, we can express our results as follows:

Corollary 1. In the even dimensional case:

$$
\begin{gathered}
\left.\frac{\operatorname{det} K}{\operatorname{det} K^{+}}\right|_{\text {even }}=(-1)^{\zeta\left(0 \mid L+m^{2}\right)}, \\
\left.\frac{\operatorname{det} K}{\sqrt{\operatorname{det}\left(L+m^{2}\right)}}\right|_{\text {even }}=(-1)^{-\frac{1}{2} \zeta\left(0 \mid L+m^{2}\right)} \cdot e^{-\frac{1}{2} a_{2 p}\left(K^{+}, K\right)} .
\end{gathered}
$$

The first of the terms in both expressions coincides with the one obtained by Asada in the mathematical literature [35] (after correction of a missprint there). Moreover, we also agree with the statement that the remaining anomaly contribution in our second result could only be given by a linear combination of Seeley-DeWitt coefficients - as these terms are excluded from the regularized definition of the zeta function determinant adopted in [35].

In the odd dimensional case, the eta function of the Laplacian undertakes the role of the zeta function, In fact, we have

\section{Corollary 2.}

$$
\begin{gathered}
\left.\frac{\operatorname{det} K}{\operatorname{det} K^{+}}\right|_{o d d}=(-1)^{-\eta(0 \mid A)} \cdot e^{-\left(\eta^{\prime}(0 \mid K)-\eta^{\prime}\left(0 \mid K^{+}\right)\right)}, \\
\left.\frac{\operatorname{det} K}{\sqrt{\operatorname{det}\left(L+m^{2}\right)}}\right|_{o d d}=(-1)^{-\frac{1}{2} \eta(0 \mid A)} \cdot e^{-\frac{1}{2}\left(\eta^{\prime}(0 \mid K)-\eta^{\prime}\left(0 \mid K^{+}\right)\right)} \cdot e^{-\frac{1}{2} a_{2 p+1}\left(K^{+}, K\right)} .
\end{gathered}
$$




\section{$7 \quad$ Some explicit examples}

Example 2. As a simple example of exact computation, let us consider the first order operator $A=P-a$, with $P=-i \partial_{\tau}$, acting on $S^{1}$, and $a$ a constant which can be interpreted as the mean value of a unidimensional periodic vector potential. We may define the domain of $A$ as consisting of periodic or antiperiodic functions with period $2 \pi$. Let us consider periodic (twisted) spinors. The antiperiodic case is recovered through the replacement $a \rightarrow a+1 / 2$. The related eta-function, for large $\operatorname{Re} s$, is

$$
\eta(s \mid A)=\sum_{n} \frac{n-a}{|n-a|}(n-a)^{-\frac{s}{2}} .
$$

The analytic extension near $s=0$ can be obtained by using the spectral theorem,

$$
\operatorname{Tr} A e^{-t A^{2}}=\sum_{n}(n-a) e^{-t(n-a)^{2}} .
$$

The Poisson resummation formula yields

$$
\operatorname{Tr} A e^{-t A^{2}}=-2\left(\frac{\pi}{t}\right)^{3 / 2} \sum_{n=1}^{\infty} n e^{-\frac{\pi^{2} n^{2}}{t}} \sin 2 \pi n a .
$$

A direct calculation gives

$$
\eta(s \mid A)=-2 \sqrt{\pi} \frac{\Gamma(1-s)}{\Gamma\left(\frac{s+1}{2}\right)} \sum_{n=1}^{\infty}(\pi n)^{2 s-1} \sin 2 \pi n a
$$

and, as a consequence, the eta-invariant reads, for example for $0<a<1$,

$$
\eta(A)=\eta(0 \mid A)=-2 \frac{1}{\pi} \sum_{n=1}^{\infty} n^{-1} \sin 2 \pi n a=2 a-1 .
$$

For a generic $a$

$$
\eta(A)=\eta(0 \mid A)==2 a-2[a]-1 .
$$

Note that this is defined modulo $k, k$ integer (i.e., it is a discontinuos and periodic function of $a$, of period 1). This result is obtained from classical Fourier analysis (see, e.g., [36]). On the other hand (and this is actually non-trivial), the result (7.5) is the same that one obtains by analytical continuation of the eta function $\eta(s \mid A)$ to $s=0$-what is clear, e.g. from the corresponding expression to be found in [37].

Furthermore, let us check the formula (3.24). For the sake of simplicity let us take $0<a<1$, but the analysis can be extended to a generic $a$. The spectral theorem gives

$$
\begin{aligned}
\operatorname{Tr}\left(\frac{A}{|A|} e^{-t|A|}\right) & =\sum_{n} \frac{n-a}{|n-a|} e^{-t|n-a|}=-e^{a t}+\left(e^{a t}-e^{-a t}\right) \sum_{n=1}^{\infty} e^{-n t} \\
& =\frac{\sinh (a-1 / 2) t}{\sinh t / 2}
\end{aligned}
$$


Thus

$$
\lim _{t \rightarrow 0} \operatorname{Tr}\left(\frac{A}{|A|} e^{-t|A|}\right)=2 a-1=\eta(0 \mid A)
$$

We can also compute

$$
\begin{aligned}
\int_{0}^{\infty} d t \frac{\sin m t}{t} \operatorname{Tr}\left(\frac{A}{|A|} e^{-t|A|}\right) & =\int_{0}^{\infty} d t \frac{\sin m t}{t} \frac{\sinh (a-1 / 2) t}{\sinh t / 2} \\
& =\arctan (\tanh (\pi m) \tan (a-1 / 2)) .
\end{aligned}
$$

In the limit $m \rightarrow \infty$ one gets $\pi(a-1 / 2)$, in complete agreement with the general result of Sec. 5. Eq. (3.25) is verified too.

Example 3. As another example, let us consider a spinor field defined on a Riemann surface of genus $g>1$, namely a topologically non trivial 2-dimensional curved manifold with constant curvature (see, for example, 38, 3]).

Being this a 2-dimensional problem, it is sufficient to deal with the spinorial Laplacian. Recall that a Riemannn surface is locally homeomorphic to the 2-dimensional hyperbolic plane $H^{2}$. Thus, making use of the half plane model for $H^{2}$, one has a metric tensor

$$
g_{\mu \nu}=y^{-2} \delta_{\mu \nu} .
$$

The covariant derivative for the 2-component spinor field is given by

$$
\nabla_{\mu} \psi=\partial_{\mu} \psi+\omega_{\mu} \psi
$$

where the spin connection is determined by the 2-bein $e_{\mu}^{a}$, namely

$$
\omega_{\mu}=\frac{1}{8} e_{a \nu} \nabla_{\mu} e_{b}^{\nu}\left[\Gamma^{a}, \Gamma^{b}\right]
$$

in which the 2-dimensional Dirac matrices are given in terms of Pauli matrices

$$
\Gamma^{1}=\sigma_{1}, \quad \quad \Gamma^{2}=\sigma_{2}, \quad \Gamma=\sigma_{3} .
$$

The Dirac operator is $A=i \gamma^{\mu} \nabla_{\mu}$, and its square $L$ reads

$$
L=-y^{2}\left(\partial_{x}^{2}+\partial_{y}^{2}\right)+i y \partial_{x} \sigma_{3}-\frac{1}{4}
$$

We shall deal with Weyl spinors, namely the ones which are eigenvectors with eigenvalues \pm 1 of $\sigma_{3}$. Recall that a spinor structure can be introduced in the following way (see for example [39, 40, 41]). Let us consider a $\tilde{\Gamma}$, containing the element -1 and such that $\tilde{\Gamma} /\{ \pm\}=\Gamma$. Here $\Gamma$ is a stricly hyperbolic discrete subgroup of $\operatorname{PSL}(2, R)$. Define a character $\chi: \tilde{\Gamma} \rightarrow\{ \pm 1\}$, $\chi(-I)=-1$. It is possible to show that there exist $2^{2 g}$ inequivalent choices for the spinor characters. The set of Weyl spinors may be identified as automorphic functions of weight \pm 1 .

The two dimensional self-adjoint operators given by

$$
D_{ \pm 1}=-y^{2}\left(\partial_{x}^{2}+\partial_{y}^{2}\right) \pm i y \partial_{x}
$$


are the related spinorial Laplacians, while the corresponding squares of the Dirac operators acting on the automorphic functions of weight \pm 1 are given by $L^{ \pm}=D_{ \pm 1}-1 / 4$, where the last constant term comes from the constant curvature, normalized to minus one, of the Riemann surface. From now on, we shall consider only Weyl spinors of weight 1.

As is known, we need the analytic continuation of the zeta function associated with the differential operator $D_{1}$. Such an analytic continuation can be obtained by means of the Selberg trace formula. In our case, it reads [39]

$$
\sum_{n=0}^{\infty} h\left(r_{n}\right)=\frac{V(F)}{2} \int_{-\infty}^{\infty} h(r) \psi_{2}(r) d r+\sum_{\wp} \sum_{k=1}^{\infty} \frac{\chi\left(P(\gamma)^{k}\right)}{S_{2}\left(k ; l_{\gamma}\right)} \hat{h}\left(k l_{\gamma}\right)
$$

where $F$ is a fundamental domain for $\Gamma, h(r)$ is an even function, analytic on a strip and such that its Fourier transform $\hat{h}$ is exponentially bounded, $\left(r_{n}\right)^{2}=\lambda_{n}-1 / 4, \lambda_{n}$ being the eigenvalues of the $D_{1}$ operator. Furthermore, $\gamma$ is an element of the conjugacy class associated with the length of the closed geodesic $l_{\gamma}, \wp$ is a set of primitive closed geodesics, and

$$
\psi_{2}(r)=\frac{1}{2 \pi} r \operatorname{coth} \pi r, \quad S_{2}\left(k ; l_{\gamma}\right)=\frac{2 \sinh \left(k l_{\gamma} / 2\right)}{l_{\gamma}},
$$

$\chi(P(\gamma))$ being the $2^{2 g}$ characters which define all topological inequivalent spinor fields.

It is convenient to work with the operator $L(\lambda)=D_{1}-\lambda$ and put $\delta^{2}=1 / 4-\lambda$. Thus, when $\delta \rightarrow 0, L(\lambda) \rightarrow L^{+}$. The final result for the analytical continuation reads

$$
\begin{aligned}
\zeta\left(s \mid L(\lambda)=\frac{V(F)}{2 \pi}\right. & {\left[\frac{\Gamma(s-1)}{\Gamma(s)} \delta^{2-2 s}+2 \int_{0}^{\infty} d r \frac{r\left(r^{2}+\delta^{2}\right)^{-s}}{e^{2 \pi r}-1}\right] } \\
+ & \frac{\sin (\pi s)}{\pi} \int_{0}^{\infty}[t(t+2 \delta)]^{-s} \frac{d}{d t} \ln Z(t+1 / 2+\delta) d t,
\end{aligned}
$$

where the Selberg zeta function is given by

$$
\left.Z_{(} s\right)=\prod_{\left\{l_{\gamma}\right\}} \prod_{k}\left[1-\chi\left(l_{\gamma}\right) e^{-(s+k) l_{\gamma}}\right] .
$$

Let us consider the massive case. Here $\delta^{2}=m^{2}$ and, from Eq. (7.17), we have

$$
\zeta\left(0 \mid L^{+}+m^{2}\right)=\frac{V(F)}{2 \pi}\left(\frac{\zeta_{R}(2)}{2 \pi}-m^{2}\right),
$$

where $\zeta_{R}(z)$ is the Riemann zeta function and

$$
\zeta^{\prime}\left(0 \mid L^{+}+m^{2}\right)=-C(m)-\ln Z(1 / 2+m),
$$

with

$$
C(\delta)=\frac{V(F)}{\pi}\left[\int_{0}^{\infty} d r r \frac{\log \left(r^{2}+\delta^{2}\right)}{e^{2 \pi r}-1}+\frac{\delta^{2}}{4}\left(1-\log \delta^{2}\right)\right] .
$$

In the massless case, zero modes may be present. Denoting by $N$ the number of zero modes of the Dirac operator and taking into account these zero modes in the evaluation of the zeta function regularized determinat of the operator $L^{+}$, one has the explicit results:

$$
\zeta\left(0 \mid L^{+}\right)=\frac{V(F)}{2 \pi} \frac{\zeta_{R}(2)}{2 \pi},
$$


and

$$
\zeta^{\prime}\left(0 \mid L^{+}\right)=-\frac{V(F)}{\pi} \zeta_{R}^{\prime}(-1)-\ln \left(\frac{Z^{(2 N)}(1 / 2)}{2 N !}\right),
$$

which show the power of our procedure.

According to the results of the previous sections, these formulas allow now for the explicit evaluation of the functional determinants of the Dirac operators $A$ and $A+i m$. Finally we would like to mention that in Refs. 42, 43] one can find other explicit examples of computation of the eta invariant related to 3-dimensional compact hyperbolic manifolds.

\section{Conclusions}

We have obtained in this paper explicit formulas for the zeta and eta-functions associated with the massless and massive Dirac operators in an arbitrary manifold without boundary, as a first step towards the calculation of the corresponding determinants, what has been done subsequently. A number of explicit examples have then been considered. We have proceeded with the calculation in complete rigor and detail, since both are necessary when trying to elucidate the long standing issues concerning the phase of the Dirac operator determinant and the usual assumptions (in the physical literature) that the determinants for plus and minus $\mathrm{im}$ are coinciding and equal on its turn to the square root of the massive Laplacian determinant.

We have here settled down these issues (in the negative), once and for all, working all the time in the zeta-function (and eta-function) domain and using both powerful mathematical theorems and explicit formulas and calculations (including resummations of the mass expansions). Our main results are given in the form of several propositions that provide clear answers to the above conjectures (see Sec. 6).

A remarkable finding is the fact that, contrary to what had been ordinarily supposed, the ambiguity present in the definition of the associated fermion functional determinant in the massless case does actually disappear in the massive case. This gives rise to a phase of the Dirac determinant that agrees with a very recent calculation in the mathematical literature (after an improvement of the last). Our very explicit results for the multiplicative anomaly of the determinants of the massive Dirac operators (with $+i m$ and $-i m$, respectively) are also in full agreement with recent calculations, in the overlapping situations. All those provide solid checks of our method and of our final formulas.

\section{Appendix}

In this Appendix, we shall consider a covenient algorithm, which is equivalent to the calculus of $\Psi$ DOs, in order to construct a parametrix for the resolvents of Dirac-like operators. We follow the classical Ref. [44] (see also [45]). For the sake of simplicity, let us consider $K=A+i m$, as a massive Dirac operator on a flat (compact) manifold. Let us introduce the $\varepsilon$-symbol

$$
\sigma_{\varepsilon}(K)=e^{i \frac{k x}{\varepsilon}} K e^{-i \frac{k x}{\varepsilon}}=K\left(x, p+\frac{k}{\varepsilon}\right),=\not p-A+\frac{\not p}{\varepsilon}+i m,
$$

where $\varepsilon$ is a real parameter, defined in order to determine recursively the parametrix. The $\varepsilon$-symbol for the resolvent is given by

$$
\left(\sigma_{\varepsilon}(K)-\frac{\lambda}{\varepsilon}\right) R_{\lambda}(\varepsilon ; x, k)=I .
$$


Here, $I$ is the identity operator and $\lambda$ a complex number. The parametrix for the symbol of the complex power is obtained putting $\varepsilon=1$. It is given by

$$
\sigma\left(K^{-s}\right)(x, k)=\frac{1}{2 \pi i} \int_{\Gamma_{\theta}} d \lambda \lambda^{-s} R_{\lambda}(x, k),
$$

where $\Gamma_{\theta}$ is a suitable cut in the $\lambda$ complex plane. Recall that the Fourier transform of (9.3) gives the parametrix for the kernel of the complex power $K^{-s}$. One may solve Eq. (9.2) with the ansatz

$$
R_{\lambda}(\varepsilon ; x, k)=\sum_{l=1}^{\infty} \varepsilon^{l} R_{l}(x, k)
$$

As a result, one has the recurrence relations

$$
(\not l-\lambda) R_{1}=I, \quad(k l-\lambda) R_{l}+K R_{l-1}=0, \quad l=2,3, \ldots .
$$

Thus, formally one obtaines

$$
\begin{aligned}
& R_{\lambda}(x, k)=\frac{1}{k^{\prime}-\lambda}\left(I-K \frac{1}{k^{\prime}-\lambda}+K \frac{1}{k^{\prime}-\lambda} K \frac{1}{k^{\prime}-\lambda}\right. \\
& \left.-K \frac{1}{k j-\lambda} K \frac{1}{k-\lambda} K \frac{1}{k j-\lambda}+\ldots\right) \text {. }
\end{aligned}
$$

The choice of the cut in the complex $\lambda$ plane depends on the meromorphic structure of $R_{\lambda}(x, k)$. If $K$ is hermitian, the poles are on the real axis and two possible inequivalent cuts can be chosen in the lower or upper complex plane, as discussed in the Introduction. If a mass is present or, in general, the operator $K$ is non-hermitian, the situation changes. As an illustration, we may consider the free case, namely $K^{(0)}=\not p+i m$. A formal resummation gives

$$
R_{\lambda}^{(0)}(x, k)=\frac{1}{k^{\prime}+i m-\lambda}=\frac{\not l-i m+\lambda}{k^{2}-(i m-\lambda)^{2}} .
$$

Here the poles are located in the upper complex plane and one is forced to choose the lower cut in Eq. (9.3). This argument is also valid in the interacting case, because the "free" contribution can be additively separated in Eq. (9.6).

On the other hand, in the case of the adjoint operator $K^{+}$, the only change is $i m \rightarrow-i m$ and it is plain that, in this case, the cut must be chosen in the upper complex $\lambda$ plane, as argued in Sec. 4.

\section{Acknowledgments}

EE is indebted with the members of the Department of Mathematics and of the Center for Theoretical Physics at MIT, specially with Dan Freedman and Robert Jaffe, for very kind hospitality during the time this work was carried out. We also thank Andrei Bytsenko for interesting discussions. This work has been supported by the cooperative agreement INFN (Italy)-DGICYT (Spain). EE has been financed also by CIRIT (Generalitat de Catalunya), grant 1998BEAI400208, and by DGICYT (Spain), project PB96-0925. 


\section{References}

[1] E. Elizalde, S. D. Odintsov, A. Romeo, A.A. Bytsenko and S. Zerbini. Zeta Regularization Techniques with Applications. World Scientific, Singapore (1994).

[2] E. Elizalde. Ten Physical applications of Spectral Zeta Functions. Springer-Verlag, Berlin (1995).

[3] A.A. Bytsenko, G. Cognola, L. Vanzo and S. Zerbini. Phys. Rep. 266, 1 (1996).

[4] B.S. DeWitt. The Dynamical Theory of Groups and Fields. Gordon and Breach, New York (1965).

[5] P. B. Gilkey. Invariance Theory, the Heat Equation and the Atiyah-Singer Index Theorem. CRC Press, Boca Raton, (1995).

[6] D.B. Ray and I.M. Singer. Ann. Math. 98, 154 (1973).

[7] S. W. Hawking. Commun. Math. Phys. 55, 133 (1977).

[8] J.S. Dowker and R. Critchley. Phys. Rev. D 13, 3224 (1976).

[9] R.T. Seeley. Am. Math. Soc. Prog. Pure Math. 10, 172 (1967).

[10] M. Wodzicki. Invent. Math. 66, 115 (1982).

[11] M. Wodzicki. Invent. Math. 75, 143 (1984).

[12] V. W. Guillemin. Adv. in Math. 55, 131 (1985).

[13] M. Atiyah, V. Patodi and I. Singer. Bull. London Math. Soc. 5, 229 (1973).

[14] R.E. Gamboa Saravi, G.L. Rossini and F.A. Schaposnik. Int. J. Mod. Phys. A 11, 2643 (1996).

[15] S. Deser, L. Griguolo and D. Seminara. Phys. Rev. D57, 7444 (1998).

[16] A. Niemi and G. Semenoff. Phys. Rev. Lett. 51, 2077 (1983); Phys. Rep. 135, 93 (1986).

[17] A. N. Redlich. Phys. Rev. Lett. 52, 18 (1984); Phys. Rev. D29, 2366 (1984).

[18] M. Wodzicki. Noncommutative Residue Chapter I. In Lecture notes in Mathematics. Yu.I. Manin, editor, volume 1289, 320. Springer-Verlag, Berlin (1987).

[19] C. Kassel. Asterisque 177, 199 (1989), Sem. Bourbaki.

[20] J. Duistermaat and V. Guillemin. Invent. Math. 29, 39 (1975).

[21] M. Kontsevich and S. Vishik. Functional Analysis on the Eve of the 21st Century. Vol. 1, 173-197 (1993).

[22] E. Elizalde. J. Phys. A 30, 2735 (1997).

[23] E. Elizalde, L. Vanzo and S. Zerbini. Commun. Math. Phys. 194, 613 (1998). 
[24] E. Elizalde, A. Filippi, L. Vanzo and S. Zerbini. Phys. Rev. D57, 7430 (1998).

[25] E. Elizalde, G. Cognola and S. Zerbini. Nucl. Phys. B532, 407 (1998).

[26] A.A. Bytsenko and F.L. Williams. J. Math. Phys. 39, 1075 (1998).

[27] G. Cognola and S. Zerbini. Lett. Math. Phys.,48, 375,(1999).

[28] V. Frolov, P. Sutton ans A. Zelnikov, The dimensional reduction anomaly Alberta-Thy-1599, hep-th/9909086 (1999).

[29] J. M. Bismut and D. S. Freed. Commun. Math. Phys. 107, 103 (1986).

[30] R.E. Gamboa Saravi, M.A. Muschietti, F.A. Schaposnik and J.E. Solomin. J. Math. Phys. 26, 2045 (1985).

[31] G. Cognola, L. Vanzo and S. Zerbini. Phys. Lett. B223, 416 (1989).

[32] A. Voros. Commun. Math. Phys. 110, 439 (1987).

[33] R. Soldati and L. Sorbo. Phys. Lett. B426, 82 (1998); D.G.C Mc Keon and C. Schubert. Phys. Lett. B440, 101 (1998); G. Dunne and T. M. Hall. Phys. Rev. D60, 065002 (1999).

[34] E. Elizalde. J. High Energy Phys., 07, 015 (1999).

[35] A. Asada. Spectral invariants and geometry of mapping spaces, in Geometric aspects of partial differential equations, B. Booss-Bavnbek and K. Wojticiechowski Eds., Contemporary Math. 242 (1999). And private communication.

[36] K. Knopp. Theorie und Andwendungen der Unendlichen Rehien. Springer, Berlin, (1931), pg. 520.

[37] A. Actor. Fortschr. Phys. 35, 793 (1987).

[38] S. Zerbini. Lett. Math. Phys. 27, 19 (1993).

[39] D.A. Hejhal. The Selberg Trace Formula for PSL(2,R). Springer-Verlag, Berlin (1976).

[40] E. D'Hoker and D.H. Phong. Commun. Math. Phys. 104, 537 (1986).

[41] P. Sarnak. Commun. Math. Phys. 110, 113 (1987).

[42] A.A. Bytsenko, L. Vanzo and S. Zerbini. Phys. Lett. B459,535 (1999).

[43] A.A. Bytsenko, A.E. Concalves and F.L. Williams. Chern-Simon Invariants of Closed Hyperbolic three Manifolds. UEL-DF-990501, hep-th/9908037 (1999).

[44] M. Atiyah, R. Bott and V. Patodi. Inv. Math. 19, 279 (1973).

[45] G. Cognola, L. Vanzo and S. Zerbini. Phys. Lett. B241, 381 (1990). 